\title{
Prognostic analysis of tumor mutation burden combined with immune infiltration of Thymic epithelial tumors
}

Pinglang Ruan

Department of Microbiology,Xiangya school of Medicine,Central South University

Dan Liu

Central South University Xiangya School of Medicine

Lili Wang

Central South University Xiangya School of Medicine

Ling Qin

Xiangya Hospital Central South University

Yurong Tan ( $\square$ yurongtan@csu.edu.cn )

Central South University Xiangya School of Medicine https://orcid.org/0000-0002-0283-2105

\section{Primary research}

Keywords: Thymic epithelial tumors, Tumor mutation burden, Immune infiltrates, Prognosis

Posted Date: October 30th, 2020

DOI: https://doi.org/10.21203/rs.3.rs-98083/v1

License: @ (1) This work is licensed under a Creative Commons Attribution 4.0 International License. Read Full License 


\section{Abstract}

Background: Thymic epithelial tumors (TETs) are uncommon neoplasms with poor prognosis and limited effective therapeutic options. This study aims to investigate the prognosis of tumor mutation burden (TMB) and the potential association with immune infiltrates in TETs.

Methods: Tumor mutation burden (TMB) was calculated using Maftools package and the samples were classified into high-TMB and lowTMB groups. Differentially expressed genes (DEGs) combined with immune cell infiltration and survival rate were analyzed between the lowTMB and high-TMB groups.

Results: Single nucleotide polymorphism (SNP) occurred more frequently than insertion or deletion, and $\mathrm{C}>\mathrm{T}$ was the most common single nucleotide variants (SNV) in TETs. The results of Kaplan-Meier curve indicated that a high TMB was associated with worse clinical outcomes of TETs. Moreover, 3 hub immune genes associated with immune infiltration were significantly associated with prognosis. Besides, the TMB-related signature (TMBRS) model based on the three hub immune genes possessed good predictive value with area under curve (AUC) 0.729 , and patients with higher TMBRS scores showed worse TETs outcomes. In addition, infiltration levels of native CD $4^{+} \mathrm{T}$ cell, activated memory $C D 4^{+} T$ cell and follicular helper T cells in low-TMB group were higher than those in high-TMB group, which were correlated positively with prognosis of TETs.

Conclusion: TETs patients with low TMB have better prognosis than those with high TMB, and TMB might affect the development of TETs by regulating immune infiltration.

\section{Introduction}

Thymic epithelial tumors (TETs) are the most common anterior mediastinal tumors. These include, thymoma and thymic carcinoma[1]. According to the WHO (2015) classification criteria, thymic epithelial tumors are classified based on the morphology of epithelial cells and its ratio to lymphocytes. TETs are divided into 6 subtypes, namely A, AB, B1, B2, B3 (thymoma) and C (thymoma)[2]. The aggressiveness and malignancy of tumors of each subtype increase sequentially. At present, surgery is the main treatment strategy for TETs, and for metastatic or incompletely resected tumors, radiotherapy and chemotherapy are the options often used in clinics [3]. To sum it all, there is no effective treatment method for advanced and recurrent TETs [4]. To this response, finding new therapy is imperative in improving the prognosis of patients with TETs.

Nowadays, immunotherapy is an emerging research approach in cancer treatment. It has become an effective method for the treatment of advanced or metastatic cancer [5].Tumor cells can survive and grow in the process of anti-tumor immune response through immune escape mechanism. However these cells can be eliminated through immunotherapy by activating and enhancing the anti-tumor immune response. Tumor immunotherapy includes immuno-cell therapy, immune checkpoint inhibitors, small molecule synthesis inhibitors, and tumor vaccines [6] and the likes. Specifically, immunotherapy has been proven to have definite effects in the treatment of various cancers such as melanoma [7] and non-small cell lung cancer [8]. Thus, immunotherapy is expected to become an alternative therapy for TETs.

Tumor mutation burden (TMB) is defined as the total number of somatic gene coding errors, base substitutions, gene insertion or deletion errors detected per million bases. From the existing literature, it has been shown that, tumors with high TMB can express more new antigens. And in doing so, these tumors are more likely to be recognized by the immune system. Therefore, tumors with high TMB are more sensitive to immunotherapy. In recent years, programmed cell death-1 (PD-1)/ programmed death-ligand 1 (PD-L1) checkpoint inhibitors therapy has opened a new chapter in the treatment of advanced TETs [9-11]. However, Rajan et al. [12] reported that, in patients with relapsed thymoma, PD-L1 inhibition has anti-tumor activity but this is accompanied by a high frequency of immune-related adverse events. Therefore, finding suitable biomarkers to improve the efficacy of immunotherapy is crucial in TMB immunotherapy.

\section{Materials And Methods}

Acquisition of data

THYM gene mutation data, transcriptome data, and clinical data were downloaded from the TCGA database (https://portal.gdc.cancer.gov/) [11]. The data of gene mutation was from arScan2 variant Aggregation and Masking include 3,839 mutated genes. The transcriptome data of RNA-seq are from 123 Thymoma patients. Clinical data including survival time and survival status were also obtained. Upon standardizing the data, all analyses were performed using R software.

Assessment of TMB for patients and prognostic analysis 
TMB is the total number of somatic gene coding errors, base substitutions, inserts, or deletions detected in a million bases. In this study, the genetic mutations were calculated in each sample by writing prescripts. The analysis was carried out through the "Maftools" package and the TMB were divided into the high-TMB group and the low-TMB group by median. Eventually, these were combined with clinical data for Kaplan-Meier analysis. The survival differences between the two groups were compared and the $p$ values of the log-rank test were calculated.

GO/KEGG analysis and Gene set enrichment analysis (GSEA)

Based on the median of TMB, we were able to have 60 low-TMB samples and 57 high-TMB samples. We then identified differentially expressed genes (DEGs) into two groups with Fold Change $(F C)=1$ and False Discovery Rate $(F D R)<0.05$. We obtained a total of 3,936 different genes, and then drew the heatmap of different genes with the "PheatMap" package (top 40). Based on these different genes, we conducted GO and KEGG analysis through "clusterProfiler", "org.Hs.eg.db", "enrichplot" and "ggplot2". We described the enrichment results from three aspects, including biological process (BP), cellular component (CC), and molecular function (MF). We further identified Genome Encyclopedia (KEGG) pathways. The results were shown with the dotplot. Finally, we used GSEA software to carry out gene set enrichment analysis of the high- and the low- TMB groups, which was downloaded at https://www.gsea-msigdb.org/gsea/index.jsp.

\section{CIBERSORT}

CIBERSORT is a newly developed silicon algorithm that uses RNA-SEQ or microarray data from a large number of samples to characterize portions of a cell subpopulation. Transcriptome profiles were normalized via "limma” package.

Based on the CIBERSORT algorithm, we obtained the estimated scores of 22 immune cell subtypes of transcriptome profiles of THYM. Moreover, we analyzed 22 immune cell infiltrates between low-and high-TMB groups.

COX analysis and risk model of hub independent risk signature

The latest immune genes were downloaded from IMMPORT database (www.immport.org/shared/home), and then intersection genes were chosen with differentially expressed genes $(\log F C>2$ and FDR $<0.05)$ through the Venn diagram. Then the expression data of the intersection genes and clinical data were merged. Univariate Cox analysis was uutilized to screen prognostic genes and multivariate Cox was utilized to further identify hub independent risk characteristics to establish risk model. The TMB related signature (TMBRS) was subsequently calculated with TMBRS $=\rrbracket$ (core $\left.{ }^{\star} \mathrm{EXPi}\right)$, where core could be derived from the multivariate Cox analysis. Receiver operating characteristic (ROC) curve was drawn to assess the predictive power of TMBRS by "survivalROC" package, and Kaplan-Meier analysis with log-rank test was obtained by"survival" package.

\section{TIMER}

We then analyzed key genes in the study by the TIMER database (https://cistrome.shinyapps.io/timer/), including prognostic genes and major mutated genes. TIMER web server is a comprehensive resource for systematical analysis of immune infiltrates across diverse cancer types [13]. The abundances of six immune infiltrates (B cells, CD4 ${ }^{+} \mathrm{T}$ cells, CD8 ${ }^{+} \mathrm{T}$ cells, Neutrophils, Macrophages, and Dendritic cells) are estimated by TIMER algorithm, including the correlation between the gene expression, mutated genes, clinical outcome or somatic cells and the abundance of immune infiltrates.

\section{Results}

Genome-wide mutation profiling in TETs

We acquired the mutation data of a total of 123 TET patients from the TCGA database. The mutation data were downloaded and visualized using the "maftools" package in R software. Waterfall plot showed higher frequency of gene mutations in patients with TET, such as GTF2I (33\%), HRAS (8\%), TTN (5\%), MUC16 (3\%), TP53 (3\%) and so on (Fig. 1A, H). In gene mutation correlation diagrams, most genes were independent and a few genes were synergistic. HRAS and GTF2I had a high synergistic correlation $(p<0.001)$ (Fig. 1B). Missense mutations were the most common type of mutation in patients with TETs (Fig. 1C), SNP occurred more frequently than insertion or deletion (Fig. 1D), and $\mathrm{C}>\mathrm{T}$ was the predominant mutation type detected (Fig. 1E). In addition, the number of mutated bases per sample was shown in Fig. 1F. In Fig. 1G, the mutation types were shown in different colors in box diagram.

Figure 1. Landscape of mutation profiles in TET samples. A, Mutation information of each gene in each sample was shown in the waterfall plot. The annotation of mutation types were shown at the bottle with various colors and the number of mutation burden was listed in the 
bar chart above the legend. B, The relationship between mutated genes C-E, Based on statistical calculations of different types of mutations, where missense mutations accounted for the majority, SNP occurred more frequently than deletion or insertion, and $\mathrm{C}>\mathrm{T}$ was the most common type of SNV. F-G, Illustration of tumor mutation burden in per samples. H, The top 10 mutated genes in TET.

TMB was associated with prognosis

In order to explore the relationship between TMB and the prognosis of patients with TETs, we downloaded the prognostic information of the patients and plotted a Kaplan-Meier curve. The results indicated that a low TMB was associated with a better clinical outcome of patients with TETs (Fig. 2).

Figure 2. Kaplan- Meier curve indicated that a low TMB was associated with a better prognosis.

Genetic changes associated with TMB and functional pathway analysis

To study the DEGs associated with TMB in TET patients, we divided the patients with TET into high TMB and low TMB groups. The heatmap showed TOP 40 DEGs in two TMB groups (Fig. 3A). The GO functional analysis revealed that, these mutant genes mainly enriched in plasma membrane signaling receptor complex, cell-cell junction, cell junction assembly and actin binding (Fig. 3B). In KEGG pathway analysis, PI3K-Akt signaling pathway, cytokine-cytokine receptor interaction, human papillomavirus infection, Rap1 signaling pathway and focal adhesion were top 5 signaling enriched (Fig. $3 C$ ). In addition, the GSEA results suggested that patients in high-TMB group tend to be more associated with tumor-related signaling pathways, including focal adhesions, ErbB signaling, ECM-receptor interaction and TGF- $\beta$ signaling pathway. (Fig. 3D).

Figure 3 Genetic changes associated with TMB and functional pathway analysis

A, The top 40 selected differentially expressed genes to be exhibited in heatmap graph with $\| \log (\mathrm{FC})>1 \mid$ and FDR $<0.05$. B-C, GO and KEGG pathway analysis for mutated genes. D, GSEA results showed the top TMB-related signaling axis, including focal adhesions, ErbB Signaling, ECM-receptor interaction and TGF- $\beta$ signaling pathway. GO, Gene Ontology; KEGG, Kyoto Encyclopedia of Genes and Genomes; TMB, tumor mutation burden.

Identification and Kaplan-Meier survival analysis of hub TMB-related immune genes

We screened 97 differential immune genes from high TMB and low TMB groups, and then utilized univariate cox analysis to screened 13 survival related genes among these 97 differentially expressed immune genes (Table 1). Then, the multivariate cox regression analysis was performed to further select 3 hub TMB-related immune genes that are highly associated with prognosis. Higher expression levels of HCK correlated positively with poor prognosis, while lower levels of CD1B and CD1E correlated with a worse prognosis (Figure,4A-C). In addition, we utilized the 3 genes to establish TMB-related signature (TMBRS) model. The AUC of ROC was 0.729 , indicating that the high predictive accuracy for our identified TMBRS model (Fig. 4D). The patients were divided into two groups according to the median value of risk score, and patients in high-risk group had poor prognosis (Fig. 4D). 
Table 1

13 prognostic mutation related genes by univariate Cox regression analysis

\begin{tabular}{|lllll|}
\hline Gene & HR & HR.95L & HR.95H & CoxPvalue \\
\hline CD1B & 0.992676 & 0.986658 & 0.99873 & 0.017808 \\
\hline CD1C & 0.97868 & 0.960149 & 0.99757 & 0.027146 \\
\hline CD1E & 0.992612 & 0.986518 & 0.998743 & 0.018253 \\
\hline HCK & 1.005474 & 1.001646 & 1.009316 & 0.005033 \\
\hline CCR9 & 0.878058 & 0.772374 & 0.998202 & 0.04687 \\
\hline RORC & 0.871029 & 0.77387 & 0.980386 & 0.022124 \\
\hline SH2D1A & 0.977006 & 0.95721 & 0.99721 & 0.025918 \\
\hline GRAP2 & 0.936357 & 0.889272 & 0.985936 & 0.012489 \\
\hline TRAJ61 & 0.313034 & 0.121074 & 0.809341 & 0.016556 \\
\hline TRAV18 & 0.247726 & 0.072607 & 0.84521 & 0.025845 \\
\hline TRBV14 & 0.858547 & 0.737851 & 0.998986 & 0.048485 \\
\hline TRBV18 & 0.893928 & 0.801581 & 0.996915 & 0.043851 \\
\hline TRBV19 & 0.941754 & 0.888898 & 0.997754 & 0.041723 \\
\hline
\end{tabular}

Figure 4 Identification and Kaplan-Meier survival analysis of hub TMB-related immune genes.

A-C, Kaplan-Meier analysis with log-rank test for identified hub TMB-related immune genes. D-E, Construction and assessment of TMBRS for TETs (the AUC of ROC curve was 0.729), where patients with higher TMBRS conferred poor survival outcomes.

Associations of 3 hub TMB-related immune genes expression with immune cells infiltration

Then multivariate Cox is utilized to further identify hub independent risk characteristics and establish a risk model. We further screened three hub survival-related genes from 13 prognostic mutation related genes to construct our prognostic model. The 3 hub TMB-related immune genes expression between the high- and the low-TMB groups were shown in volcano plot in Fig. 5A. Among these, CD1B and CD1E were lowly expressed in high-TMB group, while HCK was highly expressed. More importantly, we further assessed the underlying relationships of the expression of these hub genes with immune infiltrates in TET microenvironment. The expression of HCK was negatively correlated with the infiltration of $\mathrm{CD}^{+} \mathrm{T}$ cell and $\mathrm{CD} 4^{+} \mathrm{T}$ cell, while the levels of $\mathrm{CD} 1 \mathrm{~B}$ and $\mathrm{CD} 1 \mathrm{E}$ were positively correlated with immune infiltrates. These immune infiltrates include B cell, $\mathrm{CD}^{+} \mathrm{T}$ cell, CD4 ${ }^{+} \mathrm{T}$ cell, macrophages and dendritic cells (Fig. 5B-D). These suggest that, low-expression CD1B, CD1E and high-expression HCK in tumors can inhibit immuno-immersion.

Figure 5 Associations of 3 hub TMB-related immune genes expression with immune cells infiltration. A, Expression analysis of 3 hub TMBrelated immune genes with $\mid \log$ (Foldchange) $>1$ | and FDR $<0.05 ; \mathrm{B}-\mathrm{D}$, The relationship of 3 hub TMB-related immune genes with immune infiltrates.

Tumor infiltrating immune cells (TIICs) in high-and low-TMB groups

To investigate the correlation between TIICs and TMB in TETs, we first used CIBERSORT to calculate infiltration of 22 immune cells in the patients with TETs (Fig. 6A). Besides, the Wilcoxon rank-sum test indicated that native $\mathrm{CD}^{+}{ }^{\mathrm{T}}$ cells, plasma cells, activated memory CD ${ }^{+} \mathrm{T}$ cell, follicular helper T cells and regulatory T cells were higher infiltrating in low-TMB group, while native B cells, activated NK cells, resting mast cells, activated dendritic cells, M0, M1and M2 macrophages showed higher infiltrating levels in high-TMB group (Fig. 6B).

Figure 6 TIICs in high-and low-TMB groups. A Summary of estimated fractions of 22 immune cell subtypes from the CIBERSORT algorithm. B, TIICs associated with TMB. Red means high TMB and green means low TMB

Association of top mutated genes in TET with immune infiltrates 
GTF2I (33\%) and HRAS (8\%) are top mutated genes, which have synergistic mutation in patients with TET (Fig. 1H). The mutated GTF2I and HRAS can inhibit the infiltration levels of B cells, CD8 ${ }^{+}$T cells, CD $4^{+}$T cell and dendritic cells (Fig. 7A, B). Moreover, mutated HRAS can also inhibit the immune infiltration of macrophage (Fig. 7B). These results suggested that mutated GTF2I and HRAS might promote tumor development by inhibiting anti-tumor immune response, resulting in a poor prognosis for patients.

Figure 7 Association of top mutated genes in TET with immune infiltrates. A-B, Mutated GTF2I and HRAS can inhibit immune infiltration levels of B cells, CD8 ${ }^{+}$T cells, CD4 ${ }^{+}$T cell and dendritic cells. (P-value Significant Codes: $0 \leq * \star *<0.001 \leq * \star<0.01 \leq *<0.05 \leq .<0.1$ )

\section{Discussion}

Thymic epithelial tumors are rare tumors originating from thymic epithelial cells, which are related to many autoimmune diseases. Although they are sensitive to chemotherapy, there are almost no effective treatment methods for recurrent or refractory TETs. TMB is a new type of biomarker for cancers, which has proven efficacy in a variety of cancers, such as breast cancers, lung cancers, and colorectal cancers. Li et al. [14] found that high-TMB was associated with a worse prognosis, and it could promote tumor metastasis and development in patients with chromophobe cell carcinoma (chRCC). Zhang et al.[15] also reported that, the high-TMB patients showed poorer survival results, higher pathological stages and higher tumor grade in patients with clear renal cell carcinomas when compared with the low-TMB group. Apparently, our results showed that high-TMB patients had worse survival outcomes, which was consistent with these studies.

Another important finding of our study was that, three differentially expressed immune-related genes (CD1B, CD1E, and HCK) were identified in the high and low TMB groups. CD1B and CD1E are members of the CD1 antigen-presenting molecule family, which is specialized in presenting lipid antigens to T cells[16]. They are mainly expressed in B cells, bone marrow cells and monocytes. The activation of CD1 restricts $\mathrm{T}$ cells in vivo, leading to rapid antitumor cytotoxicity and interferon- $\gamma$ production, which could prevent tumor metastasis[17]. Bagchi et al.[18]found that CD1B-autoreactive T cells can effectively identify phospholipid antigens derived from tumors and exert antitumor immunity against $\mathrm{CD}_{1 \mathrm{~B}^{+}} \mathrm{T}$ cell lymphoma. Recently, it has been reported that the low expression of CD1B was associated with poorer biochemical recurrence-free survival in prostate cancer [19]. Moreover, CD1B could be used as a biomarker for the prognosis of breast cancer [20], and the low expression of CD1B might lead to the metastatic recurrence of breast cancer [21]. In addition, Yuan et al.[22] found that, the low expression of CD1B was related to the deterioration of lung adenocarcinoma. Compared with CD1B, CD1E is less studied, except that Yang et al. [23] found that CD1E could be used as a prognostic indicator of cervical cancer. Similar results to our study was that, CD1B and CD1E could be used as prognostic indicators of TETs, and the low expression levels might be related to the poor prognosis.

HCK is an important member of the receptor tyrosine protein kinase family and is expressed in myeloid monocytes and B lymphocytes [24]. It can regulate series of cell processes, including mitosis, differentiation, survival, migration and adhesion and the likes. In addition, HCK plays an important role in the integrin signaling pathway, which is closely related to tumor proliferation, invasion and metastasis [25]. It has been reported that, excessive activation of HCK is associated with various leukemias, such as chronic myelogenous leukemia (CML) and acute lymphoblastic leukemia, as well as solid malignancies including colorectal cancer, breast cancer and gastric cancer. In gastric tumor epithelial cells, over expression of HCK can induce alternative polarization of macrophages, activation of STAT3 and promote tumor growth [26]. Roseweir et al.[27] reported that, HCK was highly expressed in patients with colorectal cancer, it could promote tumor progression, inhibit local lymphocyte inflammatory infiltration and are related to the poor prognosis of patients. This poor prognosis is suggested to be due to effects on proliferation and facilitation of an alternative M2-like macrophage polarization. Moreover, HCK participated in the development of glioblastoma (GBM) by mediating the EMT process. This might become a promising therapeutic target for GBM [28].

In our study, we found that CD1B, CD1E and HCK were closely associated with the patient's prognosis. This may be related to its suppression of anti-tumor immune response. Therefore, a prognostic model (TMBRS) was developed using the 3 hub immune genes which can be very useful for survival prediction. Patients with high TMBRS revealed worse survival outcomes when compared with those with low TMBRS.

The tumor microenvironment, especially tumor immune infiltration affects the effects of tumor immunotherapy [29]. And interestingly, this effect plays a dual role in cancer, which can inhibit tumor growth or promote tumor growth [30].So far, there have been many studies on the relationship between immune cells and tumor immunotherapy. As the main force in tumor immunity, T cells can migrate to the tumorigenesis site at a higher rate and inhibit the survival of cancer cells[31].Once T cells (mainly activated effector cells) are reduced in tumor, this may lead to poor prognosis in tumor patients, like colon cancer, lung cancer or other cancers. As another important role of immunity, B cells also play a positive role in tumor immunity [32, 33]. However, it is worth noting that, the anti-tumor function of B cells may also be limited to some extent. After all, many studies have shown that B cells cooperate with T cells to participate in the anti-tumor effect [34].The infiltration of macrophages plays a "double-edged sword" role in development of tumors. M1 macrophages have the role of killing 
tumor cells, while M2 macrophages are shown to promote tumor growth, so the effect of macrophages in TETs is no longer discussed here. In our results, the survival rate of the high-TMB group was significantly lower than that of the low- TMB group. Considering the reasons of immune infiltration, there was no doubt that the significant decrease of $\mathrm{T}$ cell infiltration (including native $\mathrm{CD} 4^{+} \mathrm{T}$ cells, activated memory $\mathrm{CD}^{+} \mathrm{T}$ cell and follicular helper T cells were likely to be important factors influencing the prognosis. In the high-TMB group, high infiltration of B cells could theoretically improve anti-tumor immunity, but the fact was that, patients in the high-TMB group were still a manifestation of poor prognosis. Therefore, we speculated that, due to the lack of T cells, the anti-tumor effect of B cells was obviously limited.

Tumor mutations can increase the probability of immunogenicity of cancer [35]. GTF2I is a signal-induced transcription factor that responds to various extracellular signaling pathways, including B and T cell receptor, triggering the tyrosine residue to get phosphorylated. Many studies have reported that GTF2I is mutated in most thymoma, and it can potentially be used as a new therapeutic target for patients with thymoma [36, 37]. HRAS is a member of the RAS oncogene family. Gene mutations in the RAS family are often found in thymic cancer Moreover, mutant HRAS may be related to Type 1 regulatory $\mathrm{T}(\mathrm{Tr} 1)$ cells, which differentiate in response to signals involved in T cell receptor (TCR), expressing high levels of immunosuppressive cytokine IL-10, but not Foxp3, and can suppress inflammation and promote immune tolerance [38].Our results showed that GTF2I (33\%) and HRAS (8\%) had a high synergistic correlation. Although they had no differences between low TMB and high TMB groups, they may indirectly affect the process of anti-tumor immunity.

\section{Conclusion}

In conclusion, the above studies suggested that TETs patients with low TMB have better prognoses than those with high TMB, and the three hub TMB-related immune genes (CDIB, CDIE, HCK) is an effective predictive model.

\section{Abbreviations}

TETs: thymic epithelial tumors; TMB:Tumor mutation burden; SNP:single nucleotide polymorphism; SNV:single nucleotide variants; TMBRS:TMB-related signature; AUC:area under curve; PD-1:programmed cell death-1; PD-L1:programmed death-ligand 1; GSEA:gene set enrichment analysis; DEGs:differentially expressed genes; FC:Fold Change; FDR:false discovery rate; TIICs:tumor infiltrating immune cells; CML:chronic myelogenous leukemia; GBM:development of glioblastoma; Tr1 cells:Type 1 regulatory T cells; TCR:T cell receptor

\section{Declarations}

\section{Competing interests}

The authors have declared no conflicts of interest.

\section{Consent for publication}

Not applicable.

\section{Availability of data and materials}

The datasets used and/or analyzed during the current study are available from the corresponding author on reasonable request

\section{Consent for publication}

Not applicable.

\section{Ethics approval and consent to participate}

Not applicable.

\section{Funding}

This work was supported by Grant 31670121 and 31771277 from National Natural Science Foundation of China.

\section{Authors' contributions}

Pinglang Ruan and Dan Liu analyzed the data and drafted the manuscript. Lili Wang, Ling Qin, and Yurong Tan led the analytics. Yurong Tan edited the manuscript. All authors read and approved the final manuscript. 
Not applicable.

\section{References}

1. Venuta F, Anile M, Diso D, Vitolo D, Rendina EA, De Giacomo T, Francioni F, Coloni GF. Thymoma and thymic carcinoma. Eur J Cardiothorac Surg. 2010;37(1):13-25. doi:10.1016/j.ejcts.2009.05.038.

2. Travis WD, Brambilla E, Burke AP, Marx A, Nicholson AG. Introduction to The 2015 World Health Organization Classification of Tumors of the Lung, Pleura, Thymus, and Heart. J Thorac Oncol. 2015;10(9):1240-2. doi:10.1097/JT0.0000000000000663.

3. Kelly RJ, Petrini I, Rajan A, Wang Y, Giaccone G. Thymic malignancies: from clinical management to targeted therapies. J Clin Oncol. 2011;29(36):4820-7. doi:10.1200/JC0.2011.36.0487.

4. Hishida T, Nomura S, Yano M, Asamura H, Yamashita M, Ohde Y, Kondo K, Date H, Okumura M, Nagai K, Japanese Association for Research on the T. Long-term outcome and prognostic factors of surgically treated thymic carcinoma: results of 306 cases from a Japanese Nationwide Database Study. Eur J Cardiothorac Surg. 2016;49(3):835-41. doi:10.1093/ejcts/ezv239.

5. Aoun F, Rassy EE, Assi T, Albisinni S, Katan J. Advances in urothelial bladder cancer immunotherapy, dawn of a new age of treatment. Immunotherapy. 2017;9(5):451-60. doi:10.2217/imt-2017-0007.

6. Apter D, Wheeler CM, Paavonen J, Castellsague X, Garland SM, Skinner SR, Naud P, Salmeron J, Chow SN, Kitchener HC, Teixeira JC, Jaisamrarn U, Limson G, Szarewski A, Romanowski B, Aoki FY, Schwarz TF, Poppe WA, Bosch FX, Mindel A, de Sutter P, Hardt K, Zahaf T, Descamps D, Struyf F, Lehtinen M, Dubin G, Group HPS. Efficacy of human papillomavirus 16 and 18 (HPV-16/18) AS04-adjuvanted vaccine against cervical infection and precancer in young women: final event-driven analysis of the randomized, double-blind PATRICIA trial. Clin Vaccine Immunol. 2015;22(4):361-73. doi:10.1128/CVI.00591-14.

7. Ascierto PA. Immunotherapies and novel combinations: the focus of advances in the treatment of melanoma. Cancer Immunol Immunother. 2015;64(3):271-4. doi:10.1007/s00262-014-1647-3.

8. Viteri S, Gonzalez-Cao M, Barron F, Riso A, Rosell R. Results of clinical trials with anti-programmed death 1/programmed death ligand 1 inhibitors in lung cancer. Transl Lung Cancer Res. 2015;4(6):756-62. doi:10.3978/j.issn.2218-6751.2015.12.06.

9. Katsuya Y, Horinouchi H, Asao T, Kitahara S, Goto Y, Kanda S, Fujiwara Y, Nokihara H, Yamamoto N, Watanabe S, Tsuta K, Ohe Y. Expression of programmed death 1 (PD-1) and its ligand (PD-L1) in thymic epithelial tumors: Impact on treatment efficacy and alteration in expression after chemotherapy. Lung Cancer. 2016;99:4-10. doi:10.1016/j.lungcan.2016.05.007.

10. Owen D, Chu B, Lehman AM, Annamalai L, Yearley JH, Shilo K, Otterson GA. Expression Patterns, Prognostic Value, and Intratumoral Heterogeneity of PD-L1 and PD-1 in Thymoma and Thymic Carcinoma. J Thorac Oncol. 2018;13(8):1204-12. doi:10.1016/j.jtho.2018.04.013.

11. Arbour KC, Naidoo J, Steele KE, Ni A, Moreira AL, Rekhtman N, Robbins PB, Karakunnel J, Rimner A, Huang J, Riely GJ, Hellmann MD. Expression of PD-L1 and other immunotherapeutic targets in thymic epithelial tumors. Plos One. 2017;12(8):e0182665. doi:10.1371/journal.pone.0182665.

12. Rajan A, Heery CR, Thomas A, Mammen AL, Perry S, O'Sullivan Coyne G, Guha U, Berman A, Szabo E, Madan RA, Ballester LY, Pittaluga S, Donahue RN, Tsai YT, Lepone LM, Chin K, Ginty F, Sood A, Hewitt SM, Schlom J, Hassan R, Gulley JL. Efficacy and tolerability of antiprogrammed death-ligand 1 (PD-L1) antibody (Avelumab) treatment in advanced thymoma. J Immunother Cancer. 2019;7(1):269. doi:10.1186/s40425-019-0723-9.

13. Li T, Fan J, Wang B, Traugh N, Chen Q, Liu JS, Li B, Liu XS. TIMER: A Web Server for Comprehensive Analysis of Tumor-Infiltrating Immune Cells. Cancer Res. 2017;77(21):e108-10. doi:10.1158/0008-5472.CAN-17-0307.

14. Li L, Chen X, Hao L, Chen Q, Liu H, Zhou Q. (2020) Exploration of immune-related genes in high and low tumor mutation burden groups of chromophobe renal cell carcinoma. Biosci Rep 40 (7). doi:10.1042/BSR20201491.

15. Zhang C, Li Z, Qi F, Hu X, Luo J. Exploration of the relationships between tumor mutation burden with immune infiltrates in clear cell renal cell carcinoma. Ann Transl Med. 2019;7(22):648. doi:10.21037/atm.2019.10.84.

16. Brigl M, Brenner MB. CD1: antigen presentation and T cell function. Annu Rev Immunol. 2004;22:817-90. doi:10.1146/annurev.immunol.22.012703.104608.

17. Hayakawa Y, Godfrey DI, Smyth MJ. Alpha-galactosylceramide: potential immunomodulatory activity and future application. Curr Med Chem. 2004;11(2):241-52. doi:10.2174/0929867043456115.

18. Bagchi S, Li S, Wang CR. CD1b-autoreactive T cells recognize phospholipid antigens and contribute to antitumor immunity against a CD1b(+) T cell lymphoma. Oncoimmunology. 2016;5(9):e1213932. doi:10.1080/2162402X.2016.1213932.

Page 8/16 
19. Lee CH, Chen LC, Yu CC, Lin WH, Lin VC, Huang CY, Lu TL, Huang SP, Bao BY. (2019) Prognostic Value of CD1B in Localised Prostate Cancer. Int J Environ Res Public Health 16 (23). doi:10.3390/ijerph16234723.

20. Zhang Z, Li J, He T, Ding J. Bioinformatics Identified 17 Immune Genes as Prognostic Biomarkers for Breast Cancer: Application Study Based on Artificial Intelligence Algorithms. Front Oncol. 2020;10:330. doi:10.3389/fonc.2020.00330.

21. Lu WC, Xie H, Yuan C, Li JJ, Li ZY, Wu AH. Genomic landscape of the immune microenvironments of brain metastases in breast cancer. J Transl Med. 2020;18(1):327. doi:10.1186/s12967-020-02503-9.

22. Yuan J, Yuan B, Zeng L, Liu B, Chen Y, Meng X, Sun R, Lv X, Wang W, Yang S. Identification and validation of tumor microenvironmentrelated genes of prognostic value in lung adenocarcinoma. Oncol Lett. 2020;20(2):1772-80. doi:10.3892/ol.2020.11735.

23. Yang L, Yang Y, Meng M, Wang W, He S, Zhao Y, Gao H, Tang W, Liu S, Lin Z, Li L, Hou Z. Identification of prognosis-related genes in the cervical cancer immune microenvironment. Gene. 2020;766:145119. doi:10.1016/j.gene.2020.145119.

24. Roversi FM, Pericole FV, Machado-Neto JA, da Silva Santos Duarte A, Longhini AL, Corrocher FA, Palodetto B, Ferro KP, Rosa RG, Baratti MO, Verjovski-Almeida S, Traina F, Molinari A, Botta M, Saad ST. Hematopoietic cell kinase (HCK) is a potential therapeutic target for dysplastic and leukemic cells due to integration of erythropoietin/PI3K pathway and regulation of erythropoiesis: HCK in erythropoietin/PI3K pathway. Biochim Biophys Acta Mol Basis Dis. 2017;1863(2):450-61. doi:10.1016/j.bbadis.2016.11.013.

25. Seguin L, Desgrosellier JS, Weis SM, Cheresh DA. Integrins and cancer: regulators of cancer stemness, metastasis, and drug resistance. Trends Cell Biol. 2015;25(4):234-40. doi:10.1016/j.tcb.2014.12.006.

26. Poh AR, Dwyer AR, Eissmann MF, Chand AL, Baloyan D, Boon L, Murrey MW, Whitehead L, O'Brien M, Lowell CA, Putoczki TL, Pixley FJ, O'Donoghue RJJ, Ernst M. Inhibition of the SRC Kinase HCK Impairs STAT3-Dependent Gastric Tumor Growth in Mice. Cancer Immunol Res. 2020;8(4):428-35. doi:10.1158/2326-6066.CIR-19-0623.

27. Roseweir AK, Powell A, Horstman SL, Inthagard J, Park JH, McMillan DC, Horgan PG, Edwards J. Src family kinases, HCK and FGR, associate with local inflammation and tumour progression in colorectal cancer. Cell Signal. 2019;56:15-22. doi:10.1016/j.cellsig.2019.01.007.

28. Wang Z, Ying C, Zhang A, Xu H, Jiang Y, Lou M. (2020) HCK promotes glioblastoma progression by TGFbeta signaling. Biosci Rep 40 (6). doi:10.1042/BSR20200975.

29. Zhang Y, Zhang Z. The history and advances in cancer immunotherapy: understanding the characteristics of tumor-infiltrating immune cells and their therapeutic implications. Cell Mol Immunol. 2020;17(8):807-21. doi:10.1038/s41423-020-0488-6.

30. Schreiber RD, Old LJ, Smyth MJ. Cancer immunoediting: integrating immunity's roles in cancer suppression and promotion. Science. 2011;331(6024):1565-70. doi:10.1126/science.1203486.

31. Torcellan T, Hampton HR, Bailey J, Tomura M, Brink R, Chtanova T. In vivo photolabeling of tumor-infiltrating cells reveals highly regulated egress of T-cell subsets from tumors. Proc Natl Acad Sci U S A. 2017;114(22):5677-82. doi:10.1073/pnas.1618446114.

32. Wang SS, Liu W, Ly D, Xu H, Qu L, Zhang L. Tumor-infiltrating B cells: their role and application in anti-tumor immunity in lung cancer. Cell Mol Immunol. 2019;16(1):6-18. doi:10.1038/s41423-018-0027-x.

33. Punt S, Corver WE, van der Zeeuw SA, Kielbasa SM, Osse EM, Buermans HP, de Kroon CD, Jordanova ES, Gorter A. Whole-transcriptome analysis of flow-sorted cervical cancer samples reveals that B cell expressed TCL1A is correlated with improved survival. Oncotarget. 2015;6(36):38681-94. doi:10.18632/oncotarget.4526.

34. Nielsen JS, Sahota RA, Milne K, Kost SE, Nesslinger NJ, Watson PH, Nelson BH. CD20 + tumor-infiltrating lymphocytes have an atypical CD27- memory phenotype and together with CD8 + T cells promote favorable prognosis in ovarian cancer. Clin Cancer Res. 2012;18(12):3281-92. doi:10.1158/1078-0432.CCR-12-0234.

35. Goto Y. Tumor Mutation Burden: Is It Ready for the Clinic? J Clin Oncol. 2018;36(30):2978-9. doi:10.1200/JC0.2018.79.3398.

36. Higuchi R, Goto T, Hirotsu Y, Yokoyama Y, Nakagomi T, Otake S, Amemiya K, Oyama T, Mochizuki H, Omata M. (2020) Primary Driver Mutations in GTF2I Specific to the Development of Thymomas. Cancers (Basel) 12 (8). doi:10.3390/cancers12082032.

37. Kim IK, Rao G, Zhao X, Fan R, Avantaggiati ML, Wang Y, Zhang YW, Giaccone G. Mutant GTF2I induces cell transformation and metabolic alterations in thymic epithelial cells. Cell Death Differ. 2020;27(7):2263-79. doi:10.1038/s41418-020-0502-7.

38. Huang W, Solouki S, Koylass N, Zheng SG, August A. ITK signalling via the Ras/IRF4 pathway regulates the development and function of Tr1 cells. Nat Commun. 2017;8:15871. doi:10.1038/ncomms15871.

\section{Figures}


A

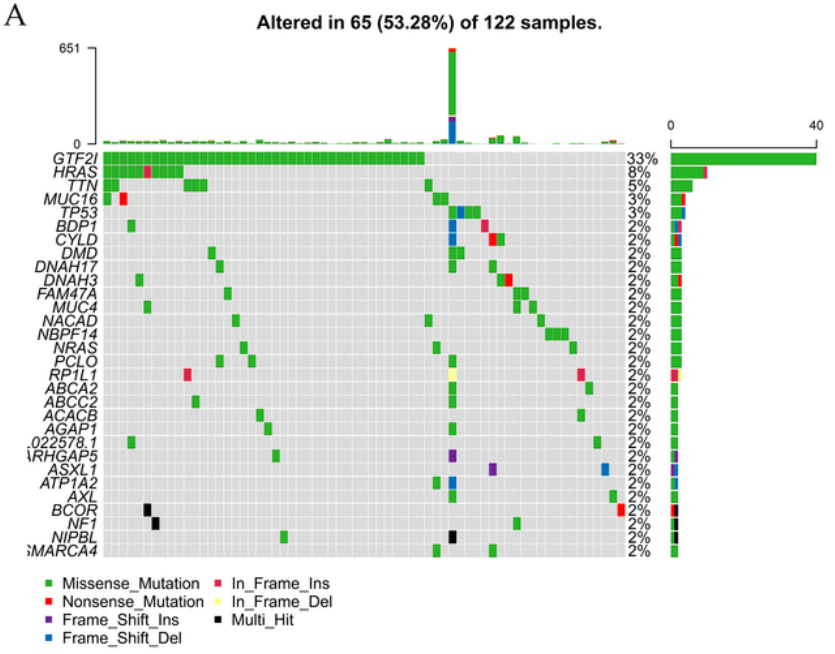

C

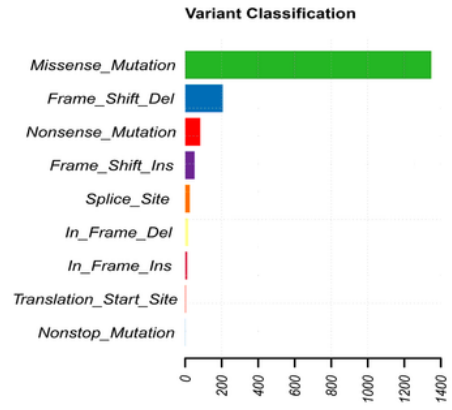

F

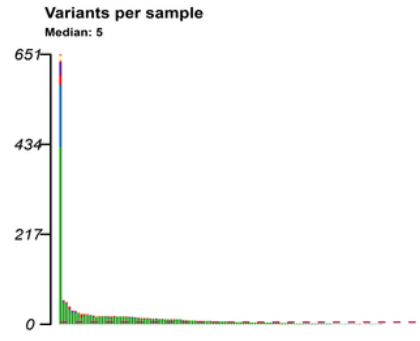

B

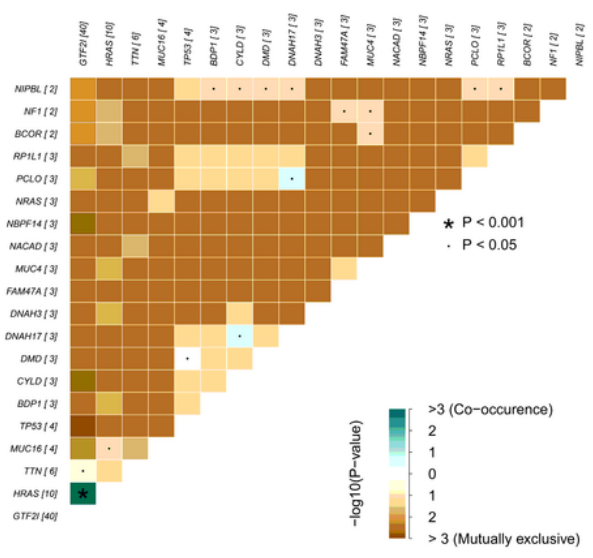

D

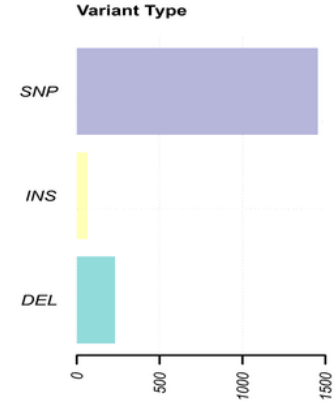

G

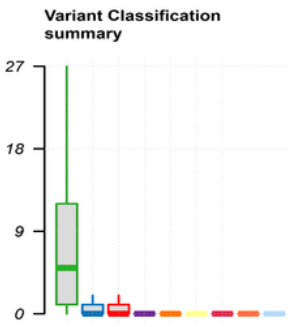

E

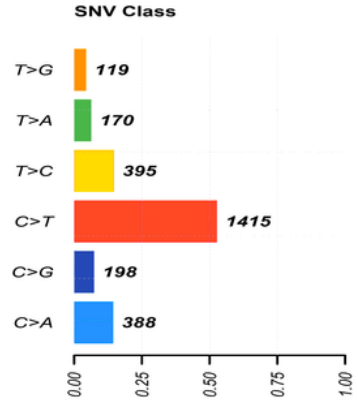

H

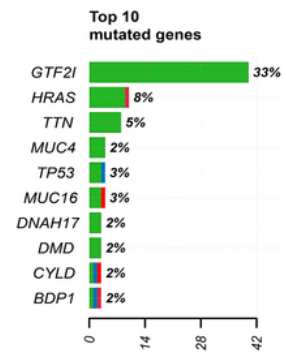

Figure 1

Landscape of mutation profiles in TET samples. A, Mutation information of each gene in each sample was shown in the waterfall plot. The annotation of mutation types were shown at the bottle with various colors and the number of mutation burden was listed in the bar chart above the legend. B, The relationship between mutated genes C-E, Based on statistical calculations of different types of mutations, where missense mutations accounted for the majority, SNP occurred more frequently than deletion or insertion, and C>T was the most common type of SNV. F-G, Illustration of tumor mutation burden in per samples. H, The top 10 mutated genes in TET. 


\section{$\operatorname{TMB}(p<0.001)$}

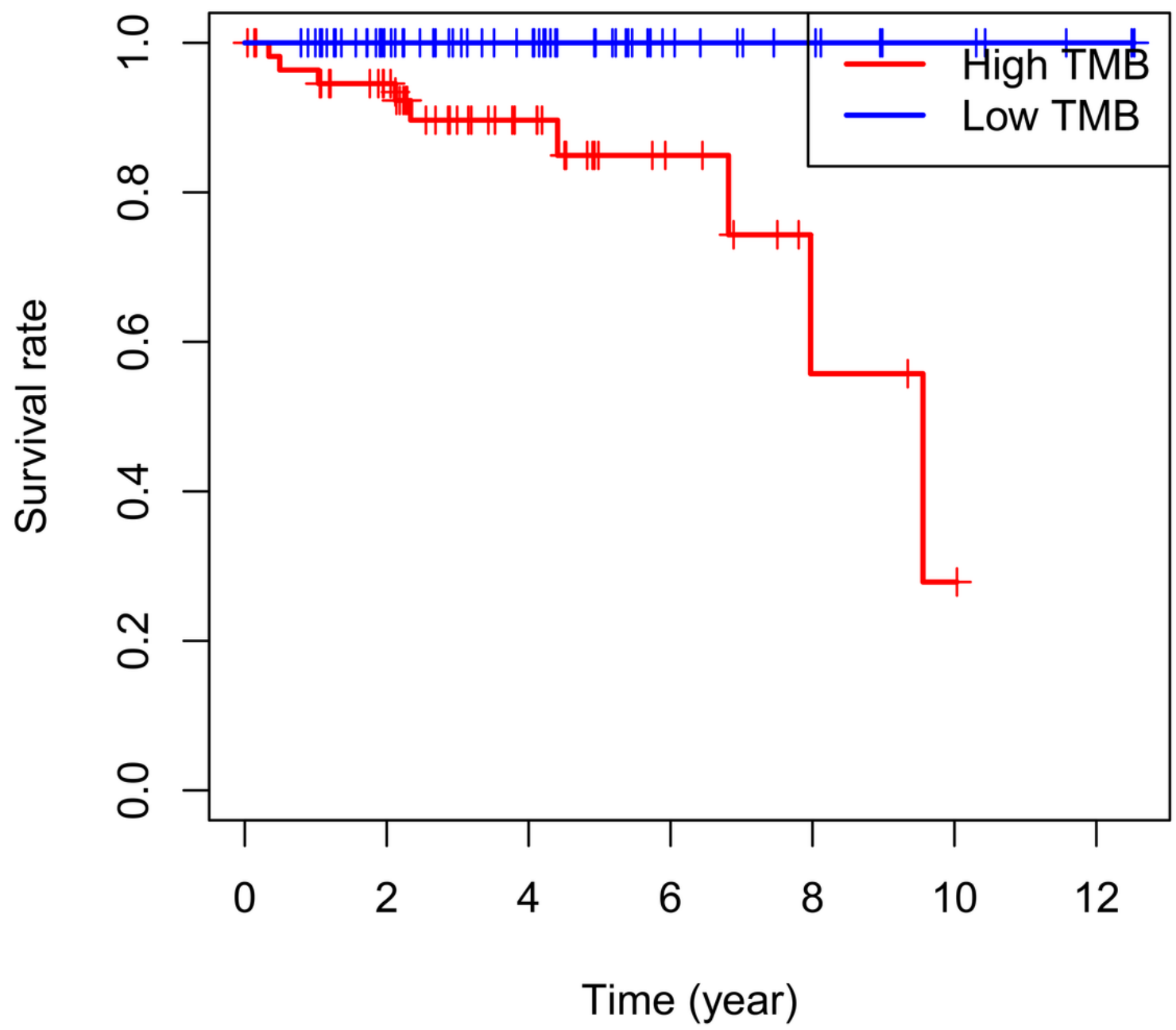

Figure 2

Kaplan- Meier curve indicated that a low TMB was associated with a better prognosis. 
A
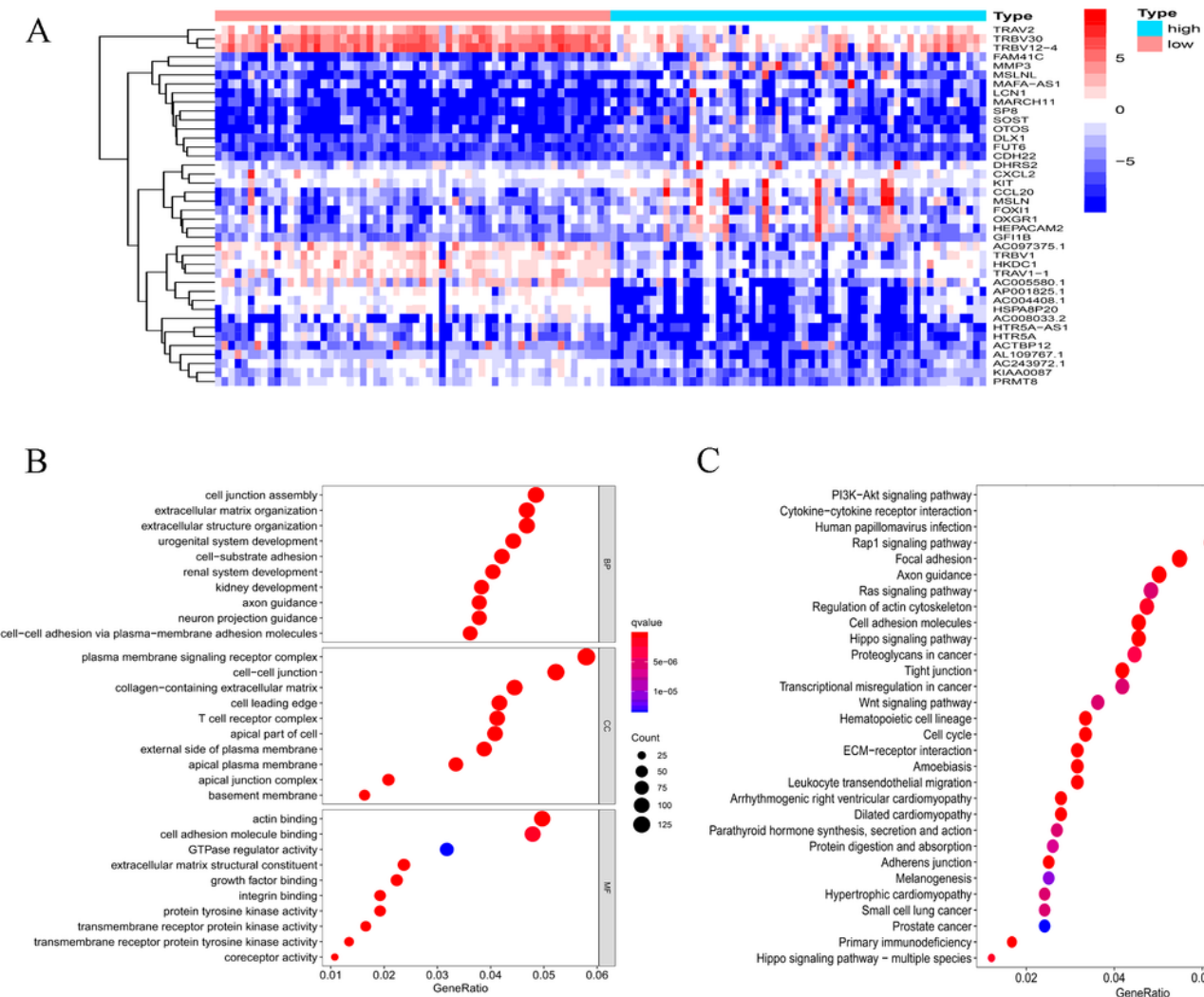

$\mathrm{C}$

$\mathrm{D}$
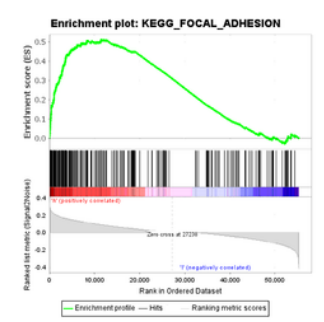
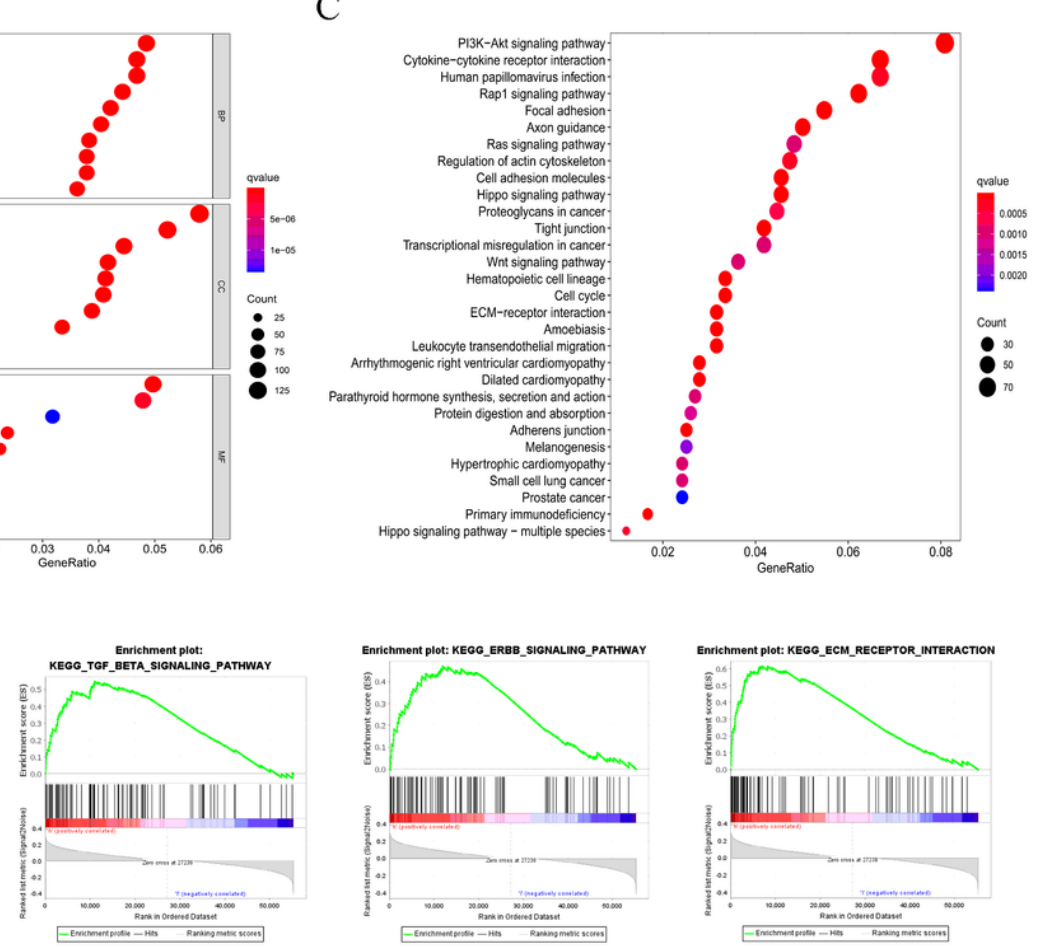

Figure 3

Genetic changes associated with TMB and functional pathway analysis A, The top 40 selected differentially expressed genes to be exhibited in heatmap graph with $\| \log (F C)>1 \mid$ and FDR $<0.05$. B-C, GO and KEGG pathway analysis for mutated genes. D, GSEA results showed the top TMB-related signaling axis, including focal adhesions, ErbB Signaling, ECM-receptor interaction and TGF- $\beta$ signaling pathway. GO, Gene Ontology; KEGG, Kyoto Encyclopedia of Genes and Genomes; TMB, tumor mutation burden. 
A

B

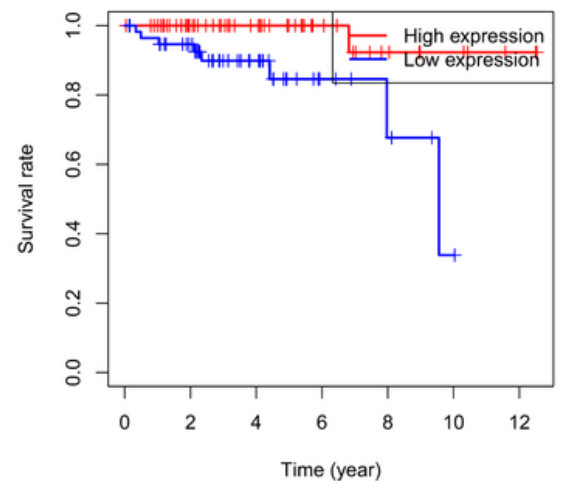

D

ROC curve $($ AUC $=0.729)$

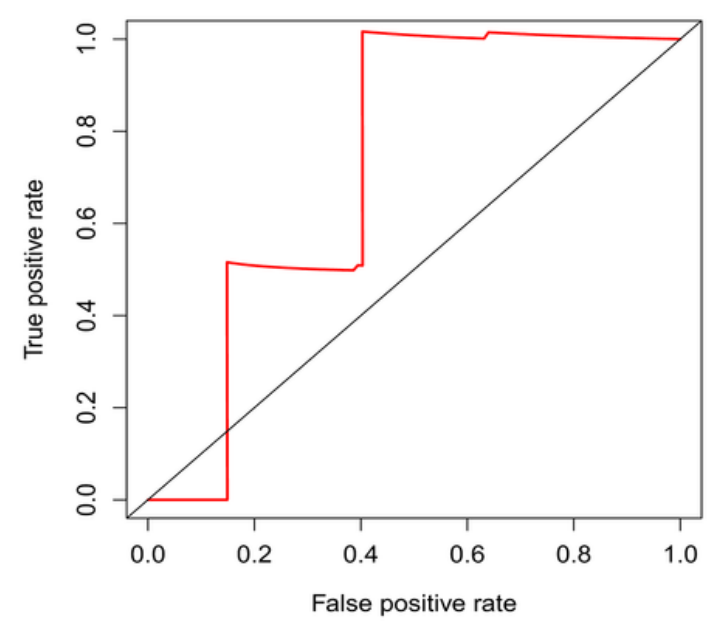

C

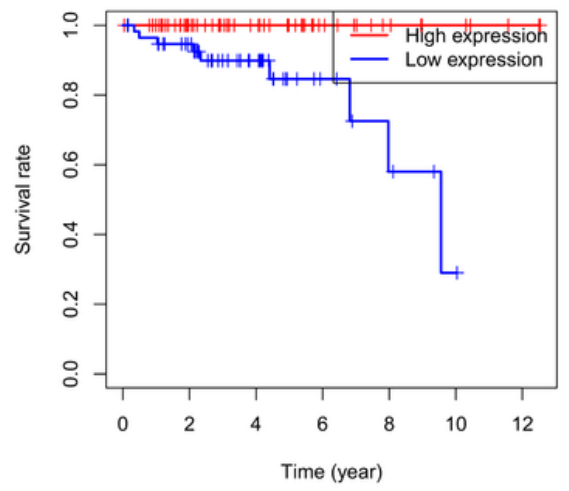

CD1E(p<0.001)
$\operatorname{HCK}(p=0.036)$

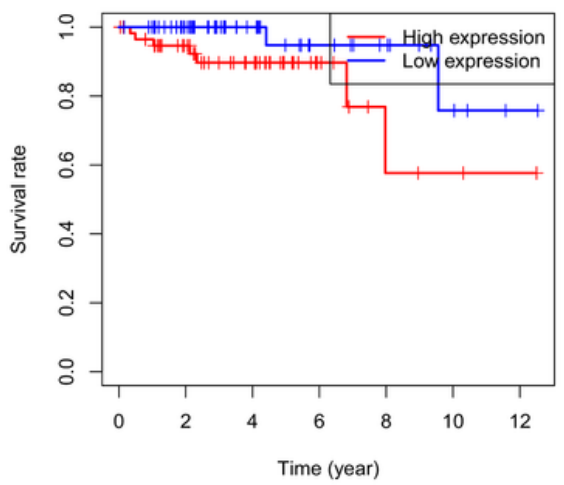

E

Survival curve $(p<0.001)$

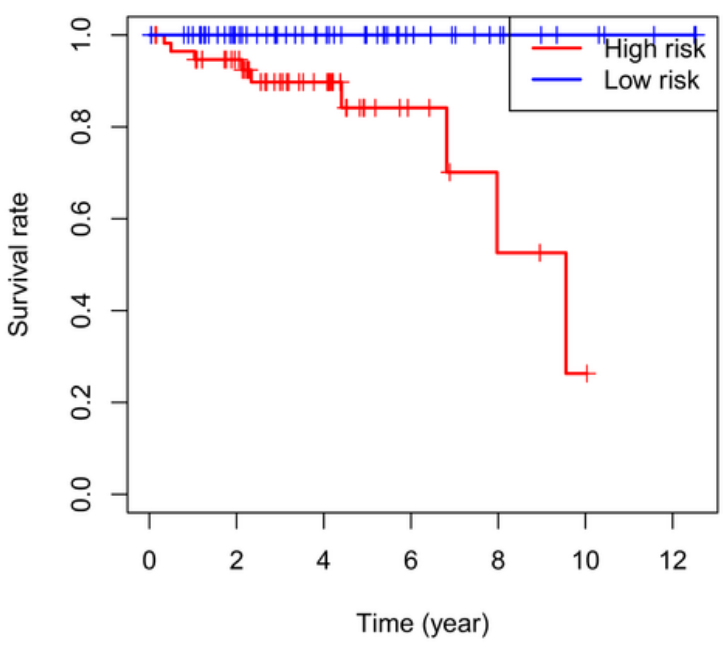

Figure 4

Identification and Kaplan-Meier survival analysis of hub TMB-related immune genes. A-C, Kaplan-Meier analysis with log-rank test for identified hub TMB-related immune genes. D-E, Construction and assessment of TMBRS for TETs (the AUC of ROC curve was 0.729), where patients with higher TMBRS conferred poor survival outcomes. 
A

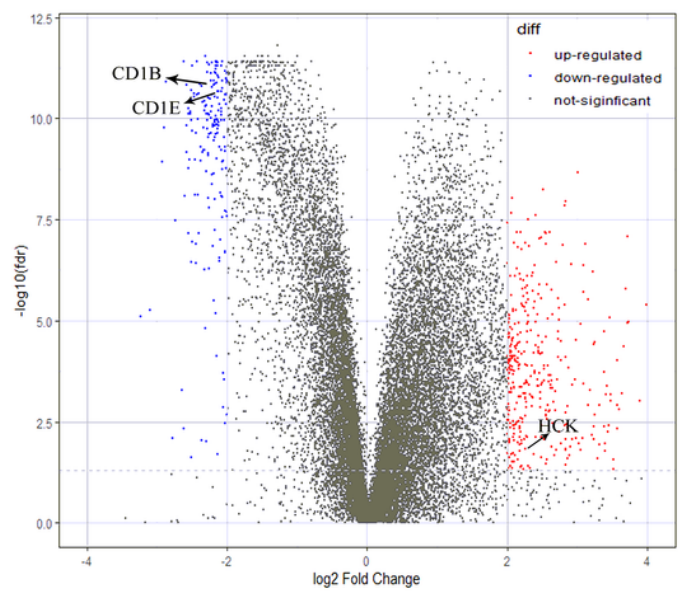

B

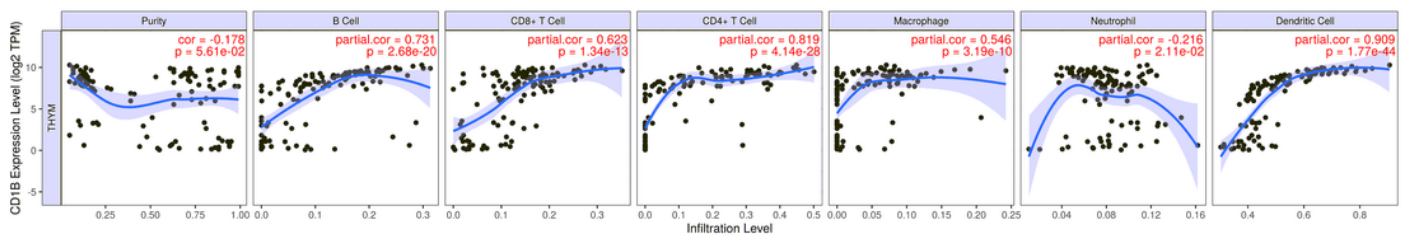

C

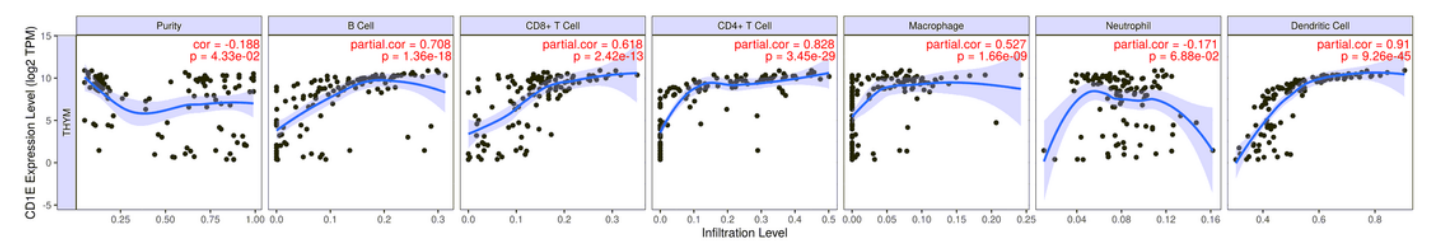

D

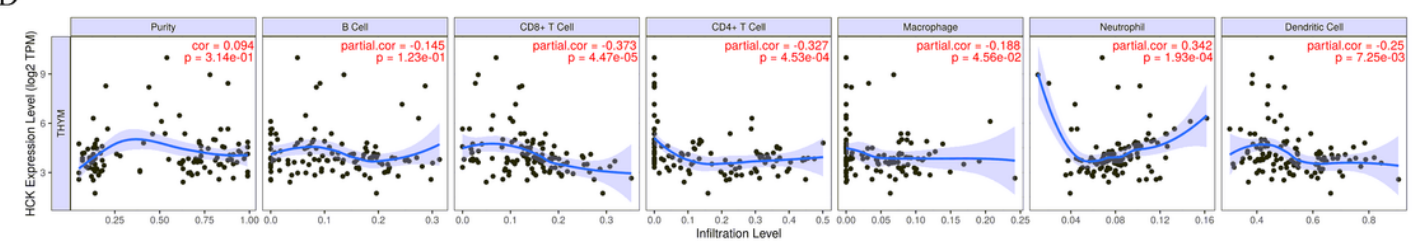

Figure 5

Associations of 3 hub TMB-related immune genes expression with immune cells infiltration. A, Expression analysis of 3 hub TMB-related immune genes with $\| \log$ (Foldchange) $>1 \mid$ and FDR $<0.05$; B-D, The relationship of 3 hub TMB-related immune genes with immune infiltrates. 
A

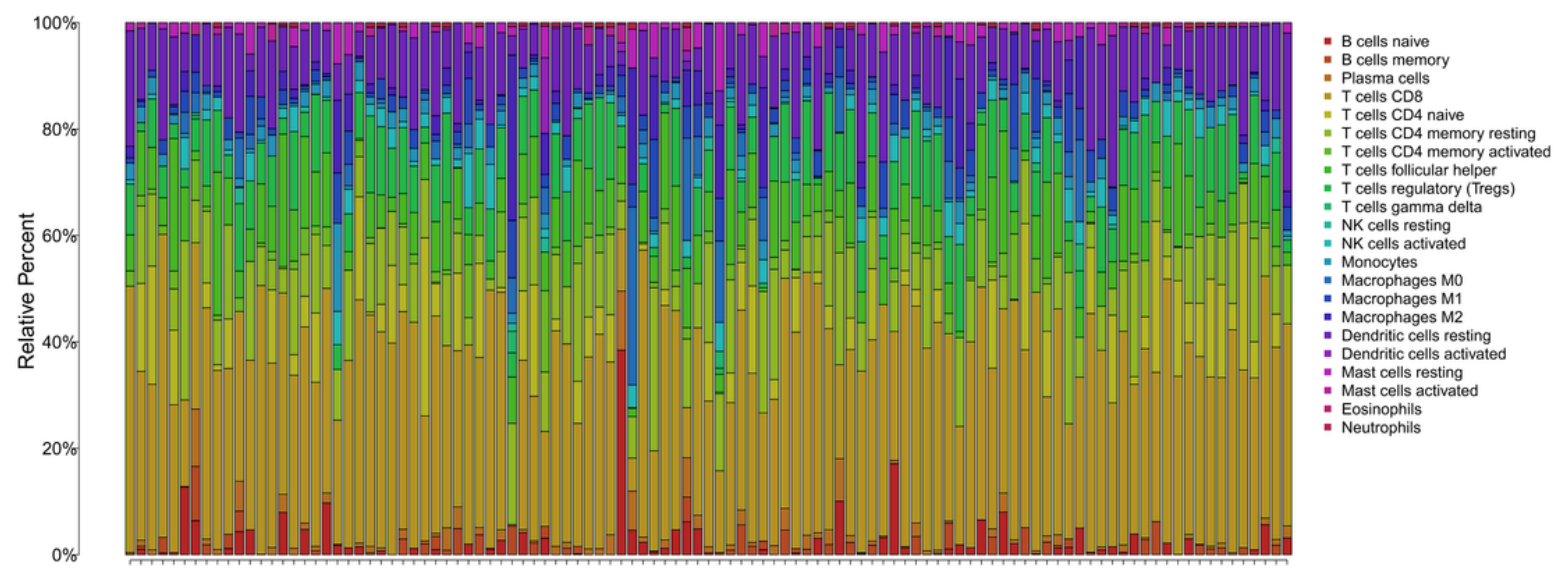

B

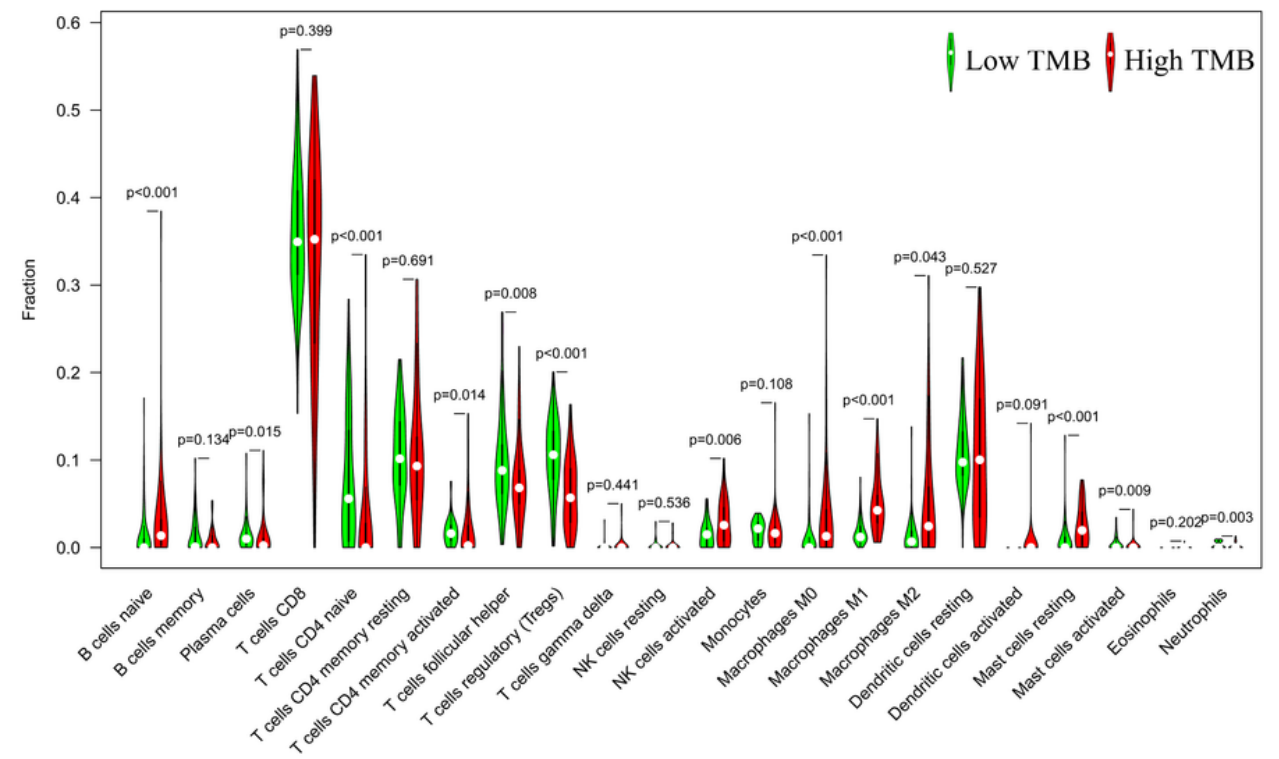

Figure 6

TIICs in high-and low-TMB groups. A Summary of estimated fractions of 22 immune cell subtypes from the CIBERSORT algorithm. B, TIICs associated with TMB. Red means high TMB and green means low TMB 
A

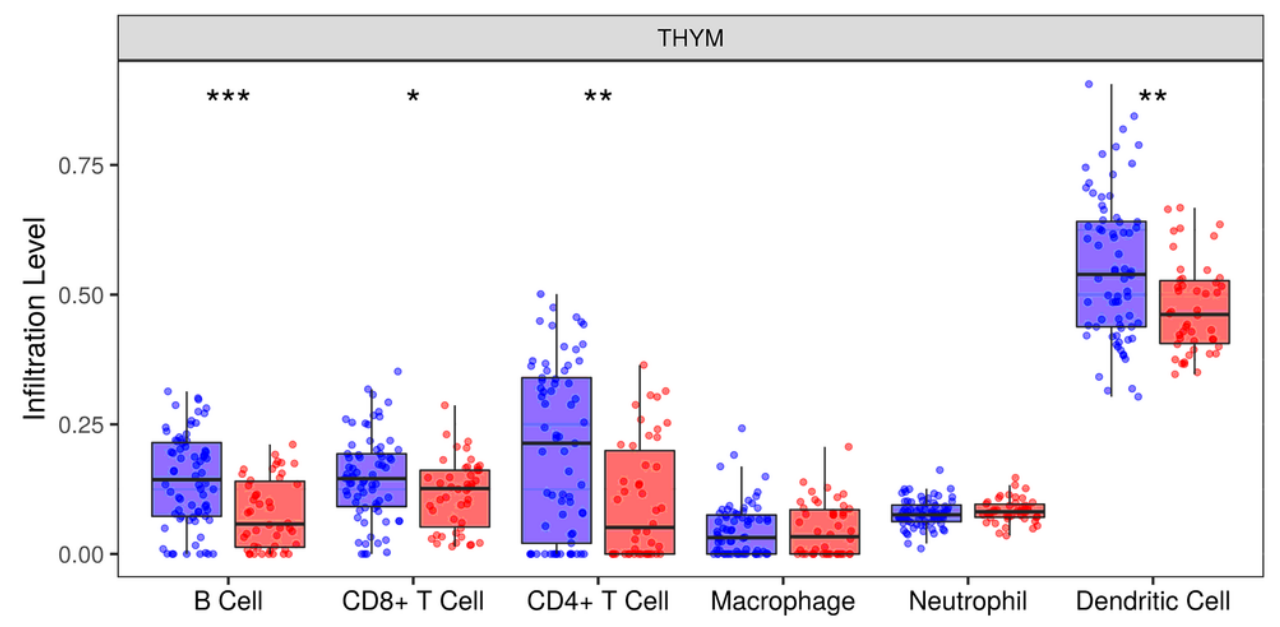

Status

审 WT GTF21

皇 Mutated GTF21

B

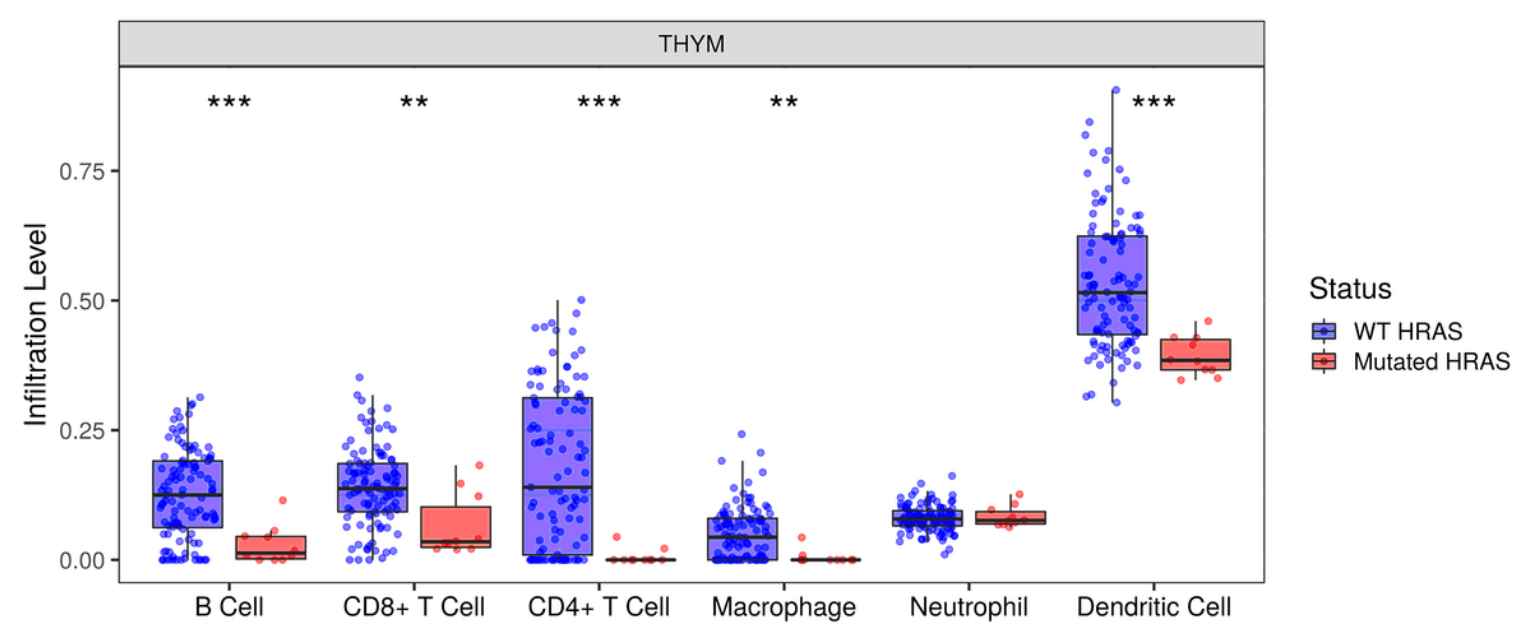

Figure 7

Association of top mutated genes in TET with immune infiltrates. A-B, Mutated GTF2I and HRAS can inhibit immune infiltration levels of B cells, CD8+ T cells, CD4+ T cell and dendritic cells. (P-value Significant Codes: $0 \leq * * * 0.001 \leq * *<0.01 \leq *<0.05 \leq .<0.1$ ) 